Research Article

\title{
Elevated hsa_circRNA_101015, hsa_circRNA_101211, and hsa_circRNA_103470 in the Human Blood: Novel Biomarkers to Early Diagnose Acute Pancreatitis
}

\author{
Chang Liu $\mathbb{D}^{1},{ }^{1}$ Xuan Zhu $\mathbb{D},{ }^{2,3}$ Xing Niu $\mathbb{D}^{4},{ }^{4}$ Lijie Chen $\mathbb{D}^{4},{ }^{4}$ and Chunlin Ge $\mathbb{D}^{2}$ \\ ${ }^{1}$ Department of General Surgery, First Affiliated Hospital of China Medical University, No. 155 Nanjing North Street, \\ Heping District, Shenyang 110001, Liaoning, China \\ ${ }^{2}$ Department of Pancreatic and Biliary Surgery, First Affiliated Hospital of China Medical University, \\ No. 155 Nanjing North Street, Heping District, Shenyang 110001, Liaoning, China \\ ${ }^{3}$ Institute of Translational Medicine, China Medical University, No. 77 Puhe Road, Shenyang North New Area, Shenyang 110122 , \\ Liaoning, China \\ ${ }^{4}$ Department of Second Clinical College, ShengJing Hospital Affiliated to China Medical University, No. 36 Sanhao Street, \\ Heping District, Shenyang 110004, Liaoning, China
}

Correspondence should be addressed to Chunlin Ge; gechunlin126@126.com

Received 10 May 2019; Revised 24 October 2019; Accepted 18 December 2019; Published 18 February 2020

Academic Editor: Kui Li

Copyright (C) 2020 Chang Liu et al. This is an open access article distributed under the Creative Commons Attribution License, which permits unrestricted use, distribution, and reproduction in any medium, provided the original work is properly cited.

\begin{abstract}
Objective. To explore potential biomarkers to accurately diagnose patients with acute pancreatitis (AP) at early stage and to auxiliary clinicians implement the best treatment options. Methods. We selected 3 patients with AP and 3 healthy controls for microarray analysis to obtain differentially expressed circular RNAs (circRNAs). To further verify the results of the microarray analysis, the six differentially expressed circRNAs were confirmed by quantitative polymerase chain reaction (qPCR). The diagnostic accuracy and sensitivity of differentially expressed circRNAs were assessed using the receiver operating characteristic (ROC) curve. A ceRNA network was constructed based on the 6 differentially expressed circRNAs. Results. There were 25 upregulated circRNAs and 26 downregulated circRNAs in the blood of patients with AP. Next, the qPCR verification results further confirmed three downregulated circRNAs, including hsa_circRNA_002532, has_circRNA_059665, and hsa_circRNA_104156, and three upregulated circRNAs including hsa_circRNA_101015, hsa_circRNA_101211, and hsa_circRNA_103470. Among them, hsa_circRNA_101015, hsa_circRNA_101211, and hsa_circRNA_103470 increased with the severity of the disease. ROC analysis showed that the three circRNA models show promise to diagnose AP. And a ceRNA network revealed that above six circRNAs could participate in complex regulated network. Conclusions. Elevated hsa_circRNA_101015, hsa_circRNA_101211, and hsa_circRNA_103470 could be used as novel biomarkers to diagnose AP patients.
\end{abstract}

\section{Introduction}

AP is one of the most common gastrointestinal diseases, and patients need hospitalization [1]. Although most patients are mild, about $20 \%$ of patients develop moderate or severe pancreatitis with surrounding pancreas tissue necrosis or multiple organ failure [2-4]. The overall mortality rate of AP patients is about $2 \%$, but close to $30 \%$ in patients with persistent organ system failure. An increased risk of pancreatic cancer in AP patients was observed in a nationwide, population-based matching cohort study [5]. Furthermore, $17 \%-22 \%$ of AP patients have the possibility of recurrence, and $8 \%-16 \%$ may develop chronic pancreatitis $[6,7]$. Therefore, early diagnosis is necessary for AP patients.

As a pancreatic inflammatory disease, AP has become one of the leading causes of gastrointestinal disease admission in the United States and many other countries. And the incidence rate demonstrates an increasing trend. There are many factors that cause the disease, such as gallstones, smoking, and alcohol abuse $[8,9]$. Of course, about $10 \%$ of patients have no cause, and they are 
idiopathic pancreatitis. The patients have usually a sudden onset with severe persistent abdominal pain, and $80 \%$ of patients are accompanied by vomiting. Pain may radiate to the back, usually in the lower chest area. Therefore, it is critical to carry out some treatments within 48 hours, such as fluid resuscitation, analgesia, and nutritional support [10]. Despite the continuous improvement in diagnostic techniques, early diagnosis is still difficult for clinicians.

Therefore, it is critical to explore early diagnostic biomarkers of AP [11]. More and more evidence has confirmed that circRNAs could play an important role in many diseases. CircRNAs are produced by reverse splicing of precursor mRNAs from exons of thousands of genes in eukaryotes [12]. At the same time, circRNAs can be secreted into blood, saliva, and other body fluids as potential biomarkers for disease prediction [13]. However, the function of most circRNAs remains largely unknown. In the ceRNA mechanism, microRNAs (miRNAs) are important posttranscriptional regulators of gene expression that act on target sites in the untranslated region of messenger RNA (mRNA) by direct base pairing. The circRNA can act as a miRNA sponge to affect the activity of miRNAs in the regulation of mRNA expression $[14,15]$. At the same time, circRNA abnormalities can lead to a variety of diseases. For example, the circMTO1 could inhibit the progression of hepatocellular carcinoma by promoting $\mathrm{p} 21$ expression, acting as a sponge of oncogenic miR-9 [16]. However, to date, no study has explored the expression of circRNAs in the blood of patients with AP. Therefore, our aim was to explore accurate biomarkers and then diagnose patients with AP as soon as possible by detecting biomarkers in the patients' blood.

\section{Materials and Methods}

2.1. Patient Information and Diagnostic Criteria. We reviewed 60 patients who were diagnosed with AP from April 2018 to September 2018 at the First Affiliated Hospital of China Medical University (Shenyang, China). In addition, we recruited 30 subjects who underwent routine health checks at the First Affiliated Hospital of China Medical University and showed no signs of disease as a control group. According to the "Guidelines for the diagnosis and treatment of acute pancreatitis (2014)," clinically meets 2 of the following 3 characteristics to diagnose AP: (1) abdominal pain consistent with AP; (2) serum amylase and/or lipase activity is at least 3 times higher than the upper limit of normal; (3) abdominal imaging examination is consistent with AP imaging changes.

In clinical treatment, mild acute pancreatitis (MAP) patients receive only relatively simple treatment, while severe acute pancreatitis (SAP) patients usually require intensive care. Therefore, we selected $30 \mathrm{MAP}$ and $30 \mathrm{SAP}$ patients to diagnose AP patients with early stage. Currently, common scoring standards are the APPACHE II scoring standard, the MCTSI scoring standard, and BISAP scoring standard. MAP diagnostic criteria were good response to fluid supplementation, without organ failure, and local or systemic complications, recovery within 1-2 weeks. And
APACHE-II score $<8$ points or the MCTSI score $<4$ points or BISAP $<2$ are MAP. Diagnostic criteria for SAP were with persistent organ failure ( $48 \mathrm{~h}$ or more). And APACHE-II score $\geq 8$ points or MCTSI score $\geq 4$ points or BISAP $\geq 2$ are SAP.

The exclusion criteria were as follows: $<18$ years of age, pregnant and lactating women, taking anticoagulant drugs, blood system diseases, tumors, liver disease, and gastrointestinal bleeding patients. The patients' information is shown in Table 1.

Then we selected 3 patients with MAP and 3 healthy participants for microarray analysis. The study was approved by the Ethics Committee of the First Affiliated Hospital of China Medical University, and informed consent was obtained from all subjects. Figure 1 depicts further experimental design details.

2.2. RNA Extraction and CircRNA Microarray Analysis. We collected whole blood from 3 MAP patients and 3 healthy participants. Total RNA from each sample was quantified using a NanoDrop ND-1000 (NanoDrop, Wilmington, DE, USA). Sample preparation and microarray hybridization were performed based on the standard protocol of Arraystar. Briefly, total RNA was digested with Rnase R (Epicenter, Inc.) to remove linear RNA and enrich for circular RNA. Then, the enriched circular RNA was amplified by a random priming method (Arraystar Super RNA Labeling Kit; Arraystar) and transcribed into fluorescent cRNA. The labeled cRNA was hybridized to Arraystar Human circRNA Array V2 $(8 \times 15 \mathrm{~K}$, Arraystar $)$. The labeled cRNA was purified by RNeasy Mini Kit (Qiagen). The concentration and specific activity of the labeled cRNA (pmol Cy3/ $\mu \mathrm{gcRNA}$ ) were measured by NanoDrop ND-1000 (NanoDrop, Wilmington, DE, USA). $1 \mu \mathrm{g}$ of each labeled cRNA was fragmented by adding $5 \mu \mathrm{l}$ of $10 \times$ blocking agent and $1 \mu \mathrm{l}$ of $25 \times$ fragmentation buffer; then the mixture was heated at $60^{\circ} \mathrm{C}$ for 30 minutes, and finally $25 \mu \mathrm{l}$ of $2 \times$ hybridization buffer was added to dilute the labeled cRNA. $50 \mu \mathrm{l}$ of the hybridization solution was dispensed into a spacer slide and assembled onto a circRNA expression microarray slide. Slides were incubated for 17 hours at $65^{\circ} \mathrm{C}$ in Agilent Hybridization Oven. The hybridization array was fixed and scanned using an Agilent Scanner G2505C wash.

Agilent Feature Extraction software (version 11.0.1.1) was utilized to analyze the acquired array images. Quantile normalization and subsequent data processing were performed using the $\mathrm{R}$ software limma package ( $\mathrm{R}$ version 3.1.2).

2.3. Differential Expression Analysis. A scatter plot is a visualization method used to assess circRNA expression variation. Differentially expressed circRNAs with statistical significance $(F C \geq 1.5$ and $P$ values $\leq 0.05)$ were identified utilizing fold change cutoffs and volcano plots, respectively. Among them, $P$ value was calculated utilizing the unpaired $t$-test. Differentially expressed 
TABLE 1: Demographic and clinical characteristics of the MAP patients, SAP patients, and the control group.

\begin{tabular}{lccrr}
\hline Characteristic & Control $(n=30)$ & MAP $(n=30)$ & SAP $(n=30)$ & $P^{\text {a }}$ \\
\hline Age $($ y) & $48.01 \pm 12.67$ & $48.26 \pm 15.24$ & $49.21 \pm 10.32$ & \\
F/M $(n)$ & $15 / 15$ & $12 / 18$ & $13 / 17$ & \\
MCTSI & & $1.4 \pm 0.93$ & $4.98 \pm 1.04$ & $<0.001$ \\
apache-ii & & $3.09 \pm 2.94$ & $9.87 \pm 4.01$ & $<0.001$ \\
BISAP & & $0.91 \pm 0.69$ & $2.64 \pm 1.01$ & $<0.001$ \\
\hline
\end{tabular}

${ }^{a}$ SAP versus control; ${ }^{b}$ SAP versus MAP.

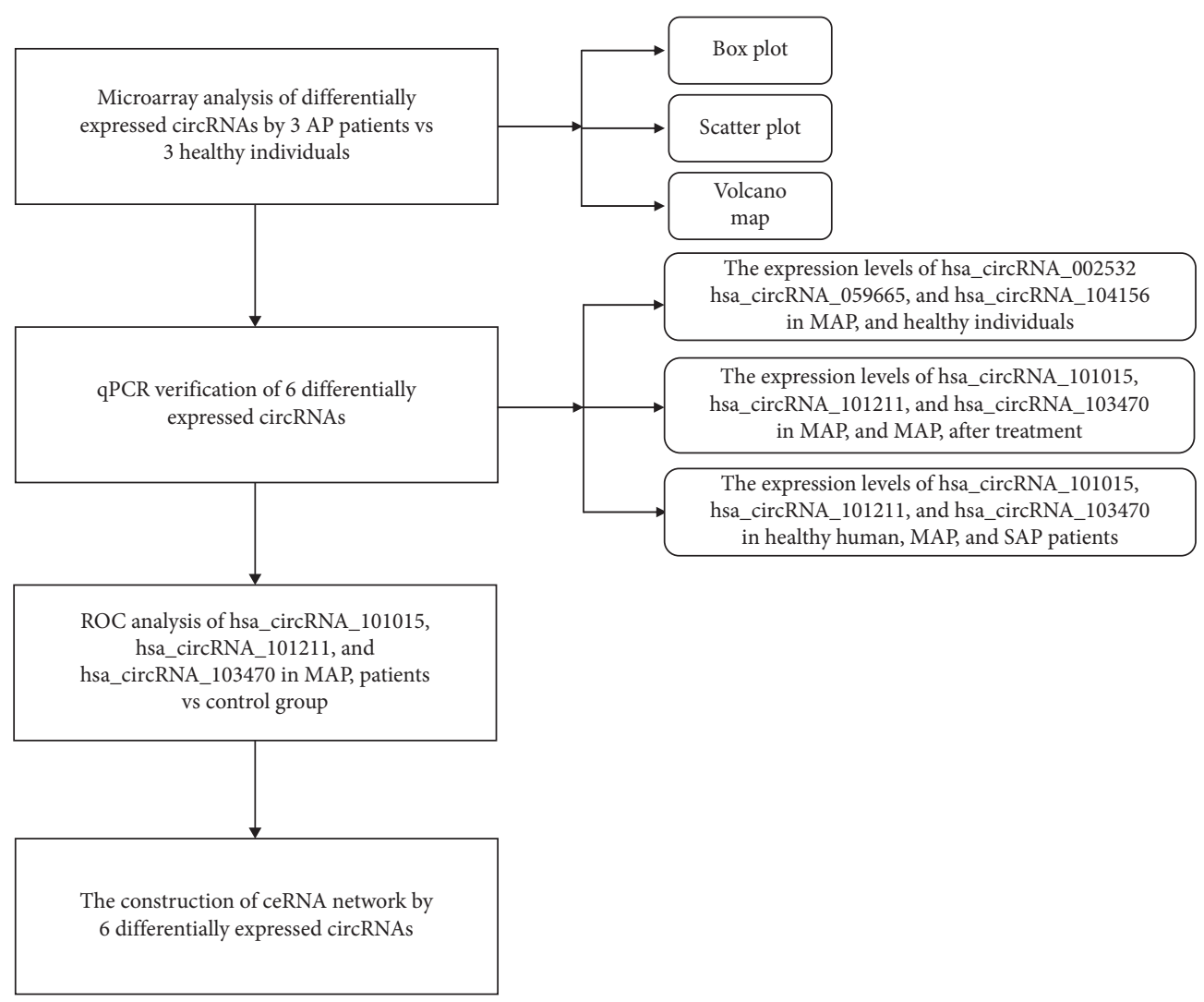

Figure 1: A flow chart of the experimental design.

circRNAs between the two samples were identified by fold change filtration. Hierarchical clustering was performed to show the distinguishable circRNA expression patterns in the samples. Shanghai Kangcheng Biological Engineering Co., Ltd. of the People's Republic of China conducted microarray work.

2.4. qPCR Verification. Total RNA was extracted from the whole blood of 30 healthy participants, 30 MAP patients, and 30 SAP patients according to standard procedures. Total RNA was reverse-transcribed into cDNA kits (Roche, Penzberg, Germany) using random primers and Transcriptor First Strand cDNA Synthesis according to the manufacturer's instructions. 6 differentially expressed circRNAs were measured by qPCR using a ViiA 7 Realtime PCR System (Applied Biosystems). The reaction conditions were as follows: $95^{\circ} \mathrm{C}$ for 10 minutes and 40 cycles of $95^{\circ} \mathrm{C}$ for 10 seconds, $60^{\circ} \mathrm{C}$ for 60 seconds. RNA levels were normalized to human $\beta$-actin. All qPCR reactions were performed in triplicate. Different primers were designed for circRNAs, rather than the more commonly used convergent primers (Figure 2). Table 2 lists all the primers.

2.5. Statistical Analysis. Statistical analyses were performed using SPSS (version 19.0; IBM, Armonk, NY, United States) and GraghPad Prism (version 7.0; GraphPad Software, La Jolla, CA, United States). The relative expression level of each circRNA was expressed by fold change and converted into $2^{-\Delta \Delta C t}$. The expression difference in circRNAs among SAP, MAP patients, and healthy individuals and between MAP patients and posttreatment serum samples was assessed using the $t$ test. To assess the diagnostic value, ROC curve was established. The cut-off value of each circRNA was analyzed by using SPSS software. Area under the ROC curves (AUCs) were calculated to evaluate the ability of the differentially expressed circRNAs. Due to the relative 


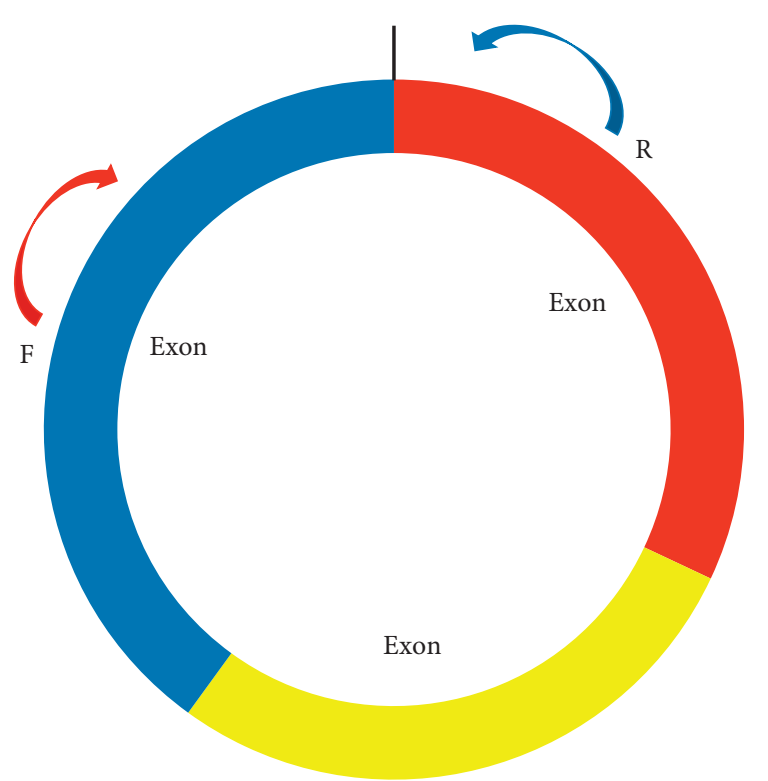

FIGURE 2: Schematic representation of polymerase chain reaction primers for specific detection of circular transcripts. Different polymerase chain reaction primers were designed for circRNA, rather than the more commonly used convergent primers.

TABle 2: Primers for circRNA and mRNA levels in qPCR.

\begin{tabular}{|c|c|c|}
\hline Target ID & Primer sequence, $5^{\prime}-3^{\prime}$ & Product size in bp \\
\hline$\beta$-actin (human) & $\begin{array}{c}\text { F: } 5^{\prime} \text { GTGGCCGAGGACTTTGATTG 3' } \\
\text { R: } 5^{\prime} \text { CCTGTAACAACGCATCTCATATT } 3^{\prime}\end{array}$ & 73 \\
\hline hsa_circRNA_002532 & $\begin{array}{l}\text { F: } 5^{\prime} \text { TGGGAGTTTTCTGCTGATGAT } 3^{\prime} \\
\text { R: } 5^{\prime} \text { GGGTTTCTTTCTCATCTCTCTCA } 3^{\prime}\end{array}$ & 119 \\
\hline hsa_circRNA_101015 & $\begin{array}{l}\text { F: 5' TATTGCCTTAGATCCTTCAAGTG 3' } \\
\text { R: } 5^{\prime} \text { TAGCATCAACCAATCGCAAGT } 3^{\prime}\end{array}$ & 78 \\
\hline hsa_circRNA_101211 & $\begin{array}{c}\text { F: } 5^{\prime} \text { TGGTGGACGCATTTTCAGC 3' } \\
\text { R: } 5^{\prime} \text { TCAGCACTTTGGTTAATCTTTCA } 3^{\prime}\end{array}$ & 87 \\
\hline hsa_circRNA_103470 & $\begin{array}{l}\text { F: 5' TAACACGCTGGCCCATTACAAG 3' } \\
\text { R: } 5^{\prime} \text { GTCCAGGTCCCGAAGGATGTAG 3' }\end{array}$ & 69 \\
\hline hsa_circRNA_059665 & $\begin{array}{l}\text { F: } 5^{\prime} \text { AGACGCCTCCAGATGCCCTT 3' } \\
\text { R: } 5^{\prime} \text { ACCAGACTGCAGGGACGGTGT 3' }\end{array}$ & 103 \\
\hline hsa_circRNA_104156 & $\begin{array}{l}\text { F: 5' AGAACTTCCTCGCACTGTGA 3' } \\
\text { R: } 5^{\prime} \text { GGGAGCTATGTGAACGAACG 3' }\end{array}$ & 83 \\
\hline
\end{tabular}

small sample size for ROC analysis, the statistical power was calculated using PASS (version 15.0), under the following conditions: $\alpha=0.05$, AUC $0=0.5$, and $n=30 . P$ value $<0.05$ was considered statistically significant.

2.6. The Construction of ceRNA Network. By combining cotargeted miRNAs, we constructed a ceRNA network by cytoscape package of $\mathrm{R}$ language $[17,18]$. In addition to measuring the number of common miRNAs, each ceRNA pair was subjected to a hypergeometric test, which was defined by four parameters: (i) $N$ is the total number of miRNAs used to predict the target; (ii) $K$ is the The number of miRNAs interacting with the selected gene; (iii) $n$ is the number of miRNAs that interact with the candidate ceRNA of the selected gene; (iv) the miRNA number common between the two genes [19]. This test used the following formula to calculate the $P$ value:

$$
P=\sum_{i=c}^{\min (K, n)} \frac{\left[\begin{array}{l}
K \\
i
\end{array}\right]\left[\begin{array}{l}
N-K \\
n-i
\end{array}\right]}{\left[\begin{array}{l}
N \\
n
\end{array}\right]}
$$

\section{Results}

3.1. Identification of Differentially Expressed CircRNAs. To investigate the expression profile of circRNAs in AP, we used microarray analysis to perform circRNA expression profiling in the blood of patients with MAP and matched normal humans. The box plot visualized the dataset distribution of circRNAs. After normalization, Figure 3(a) depicts that the $\log 2$ ratios in the three pairs of samples are almost identical. At the same time, we used a scatter plot of 


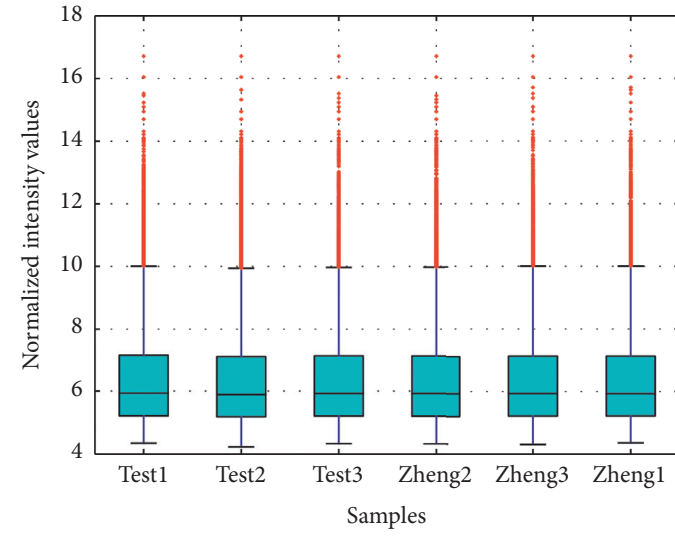

(a)

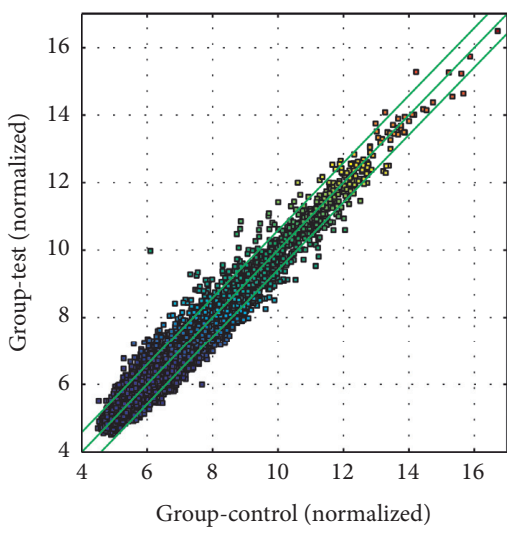

(b)

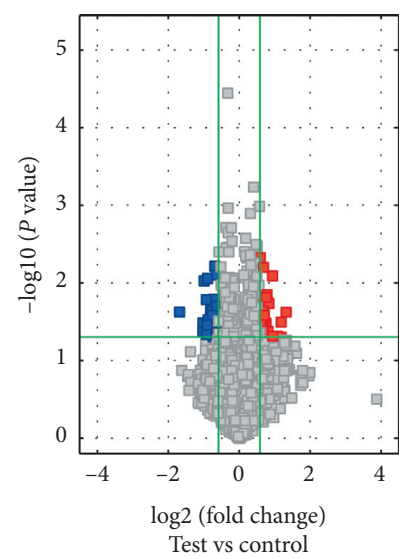

(c)

FIgURE 3: Overview of microarray features. (a) Box plots are datasets that quickly visualize circRNAs spectra (zheng: normal person's blood; test: blood of patients with acute pancreatitis). (b) Scatter plot showing circRNA expression variation between blood samples from patients with AP and matched normal persons. (c) Volcano maps of differentially expressed circRNAs.

TABLE 3: 25 upregulated in patients with AP (FDR, false discover rate; FC, fold change).

\begin{tabular}{|c|c|c|c|c|c|c|c|}
\hline CircRNA & Alias & $P$ value & FDR & FC (abs) & Chrom & best_transcript & Gene symbol \\
\hline hsa_circRNA_001747 & hsa_circ_0000246 & 0.014721747 & 0.999852393 & 1.5883102 & chr10 & uc009xqp.1 & $\mathrm{MCU}$ \\
\hline hsa_circRNA_000761 & hsa_circ_0000761 & 0.032659244 & 0.999852393 & 1.6521444 & & NM_032875 & FBXL20 \\
\hline hsa_circRNA_092519 & hsa_circ_0001233 & 0.031884015 & 0.999852393 & 1.6043929 & $\operatorname{chr} 22$ & NM_020831 & MKL1 \\
\hline hsa_circRNA_102674 & hsa_circ_0053932 & 0.004725614 & 0.999852393 & 1.5060131 & & NM_000627 & LTBP1 \\
\hline hsa_circRNA_020354 & hsa_circ_0020354 & 0.016055616 & 0.999852393 & 1.5389787 & $\operatorname{chr} 10$ & NM_001329 & СТВP2 \\
\hline hsa_circRNA_059060 & hsa_circ_0059060 & 0.020943584 & 0.999852393 & 1.6047327 & & & SEPT2 \\
\hline hsa_circRNA_074183 & hsa_circ_0074183 & 0.038344155 & 0.999852393 & 1.531 & $\operatorname{chr} 5$ & 39 & MATR3 \\
\hline hsa_circRNA_101015 & hsa_circ_0000378 & 0.023 & 0.9998 & & & & \\
\hline hsa_circRNA_100089 & hsa_circ_0010501 & 0.017554998 & 0.999852393 & 1.7280045 & & 1397 & ECE1 \\
\hline hsa_circRNA_101386 & hsa_circ_0004008 & 0.033240163 & 0.999852393 & 1.68 & & 32 & PCNX \\
\hline hsa_circRNA_101788 & hsa_circ_0038872 & 0.036 & 0.99985 & & & & $\mathrm{ATH}$ \\
\hline hsa_circRNA_103470 & hsa_circ_00e & 0.04 & 0.9998 & 2.266 & & & PLX \\
\hline hsa_circRNA_006296 & hsa_circ_0006296 & 0.0490 & 0.99985 & 2.11451 & & & \\
\hline hsa_circRNA_073748 & hsa_circ_0073748 & 0.04 & 0.9998 & 1.7 & & 3 & LMI \\
\hline hsa_circRNA_100891 & hsa_circ_0023685 & 0.006 & 0.9998 & & ch & & PAK1 \\
\hline hsa_circRNA_404746 & & 0.036 & 0.9998 & 1.5 & & $\mathrm{~N}$ & CSGALNACT2 \\
\hline hsa_circRNA_005899 & hsa_circ_00 & 0.040 & 0.9998 & 1.5 & & $\mathrm{NI}$ & LYST \\
\hline hsa_circRNA_101584 & hsa_circ_0036200 & 0.018409954 & 0.999852393 & 1.7862666 & chr15 & NM_002 & PKM \\
\hline hsa_circRNA_103448 & & 0.014194703 & 0.999852393 & & & & \\
\hline hsa_circRNA_004077 & hsa_circ_00 & 0.008 & 0.99 & & & $\mathrm{NM}_{-}$ & VAT1L \\
\hline hsa_circRNA_001363 & hsa_circ_0000172 & 0.031341076 & 0.999852393 & 1.5314787 & chr1 & ENST00000340006 & CSRP1 \\
\hline hsa_circRNA_081881 & hsa_circ_0081881 & 0.015325331 & 0.999852393 & 1.6956771 & chr7 & NM_002736 & PRKAR2B \\
\hline 007037 & hsa_circ_0007037 & 0.023032396 & 0.999852393 & 1.5823312 & & NM_024617 & ZCCHC6 \\
\hline hsa_circRNA_101211 & hsa_circ_0029407 & 0.03202411 & 0.999852393 & 2.2688595 & & & GLT1D1 \\
\hline hsa_circRNA_101833 & hsa_circ_0039908 & 0.04884264 & 0.999852393 & 1.9258456 & $\operatorname{chr} 16$ & NM_017803 & DUS2 \\
\hline hsa_circRNA_100868 & hsa_circ_0023255 & 0.026079049 & 0.999852393 & 1.6328345 & chr11 & NM_001876 & CPT1A \\
\hline
\end{tabular}

the circRNA expression profile to evaluate the change between the two groups (Figure 3(b)), where the values of the $X$ and $Y$ axes are the average normalized signal values ( $\log 2$ scaling) of the sample set. And the green line is the fold line, and the circRNA above the top green line and below the bottom green line indicates that the circRNA change between the two samples is more than 1.5 times. Next, volcano maps were used to identify differentially expressed circRNAs that were statistically significant between the two groups and are shown in Figure 3(c). Among them, the vertical lines correspond to 1.5 times up and down, and the horizontal lines represent $P=0.05$. The red dot indicates upregulated circRNAs while blue represents downregulated circRNAs. We found 51 differentially expressed circRNAs in patients with MAP $(\mathrm{FC} \geq 1.5$ and $P$ values $\leq 0.05$ ) compared with normal subjects. Among them, Table 3 shows 25 upregulated circRNAs and 26 downregulated circRNAs are described in Table 4. Next, 
TABLE 4: 26 downregulated circRNAs in patients with AP (FDR, false discover rate; FC, fold change).

\begin{tabular}{|c|c|c|c|c|c|c|c|}
\hline CircRNA & Alias & $P$ value & FDR & $\mathrm{FC}$ (abs) & Chrom & best_transcript & Gene symbol \\
\hline hsa_circRNA_102783 & hsa_circ_0007532 & 0.006107722 & 0.999852393 & 1.6128104 & chr2 & NM_015341 & NCAPH \\
\hline hsa_circRNA_101147 & hsa_circ_0028255 & 0.04131669 & 0.999852393 & 1.6696469 & chr12 & NM_002973 & ATXN2 \\
\hline hsa_circRNA_407250 & & 0.047617993 & 0.999852393 & 1.668651 & $\operatorname{chr} 9$ & NM_004269 & MED27 \\
\hline hsa_circRNA_104923 & hsa_circ_0002303 & 0.029109485 & 0.999852393 & 1.545763 & $\operatorname{chr} 9$ & NM_001006617 & MAPKAP1 \\
\hline hsa_circRNA_003912 & hsa_circ_0003912 & 0.007972547 & 0.999852393 & 1.5662106 & $\operatorname{chr} 19$ & NM_001352 & DBP \\
\hline hsa_circRNA_406493 & & 0.035076882 & 0.999852393 & & & NST000005081 & LIN54 \\
\hline hsa_circRNA_104156 & hsa_circ_0001626 & 0.009445502 & 0.999852393 & 1.9 & chr6 & $\mathrm{N}$ & $\mathrm{BACH} 2$ \\
\hline hsa_circRNA_100485 & hsa_circ_0 & 0.0304 & 0.999852393 & & chr1 & & PCNXL2 \\
\hline hsa_circRNA_102288 & hsa_circ_0046702 & 0.022518826 & 0.999852393 & 1.7462888 & $\operatorname{chr} 18$ & & YES1 \\
\hline hsa_circRNA_003910 & hsa_circ_0003910 & 0.0328 & 0.999852393 & 2.03 & $\operatorname{chr} 3$ & FNSTRO & SUMF1 \\
\hline hsa_circRNA_0c & hsa_circ_0000756 & 0.02 & 0.9998 & & $\operatorname{chr} 17$ & & $\mathrm{AT}$ \\
\hline hsa_circRNA_0 & hsa_circ_0 & & 0.999 & & & & 12 \\
\hline hsa_c & hsa_circ_0006916 & 0.01 & 0.9998 & & & & HOMER1 \\
\hline hsa_circRNA_101495 & hsa_circ_0003838 & 0.025 & 0.9998 & 1.5 & chi & 00 & TTBK2 \\
\hline hsa_circRNA_002149 & hsa_circ_0001627 & 0.00 & 0.9998 & 1.8 & chr6 & ENST00000 & $\mathrm{BACH} 2$ \\
\hline hsa_circRNA_405389 & & 0.0 & 0.999 & 1.5 & & NM_004 & $\mathrm{CO}$ \\
\hline hsa_circRNA_101899 & hsa_circ_00 & 0.0428 & 0.9998 & 2.0 & & 66 & KLHDC4 \\
\hline hsa_circRNA_103772 & hsa_circ_0008910 & 0.048867847 & 0.999852393 & 1.6283164 & & NM_001921 & DCTD \\
\hline hsa_circRNA_001124 & hsa_circ_0001124 & & & & & & \\
\hline hsa_circRNA_00 & hsa_circ_0 & 0.02 & & & & NM_01 & RCAN3 \\
\hline hsa_circRNA_002532 & hsa_circ_0002532 & 0.029871425 & 0.999852393 & 1.8331005 & $\operatorname{chrX}$ & TCONS_00017187 & XLOC_008000 \\
\hline hsa_circRNA_102306 & hsa_circ_0046995 & 0.016459014 & 0.999852393 & 1.9022111 & & NM_032142 & CEP192 \\
\hline NA_048000 & hsa_circ_0048000 & 0.04965674 & 0.999852393 & 1.6658 & & & АТР9B \\
\hline hsa_circRNA_027904 & hsa_circ_0027904 & 0.02618334 & 0.999852393 & 1.6835986 & $\operatorname{chr} 12$ & NM_020244 & CHPT1 \\
\hline hsa_circRNA_101898 & hsa_circ_0040786 & 0.035050956 & 0.999852393 & 1.8735586 & $\operatorname{chr} 16$ & NM_017566 & KLHDC4 \\
\hline hsa_circRNA_100993 & hsa_circ_0002484 & 0.032886032 & 0.999852393 & 1.519562 & & NM_014155 & ZBTB44 \\
\hline hsa_circRNA_040792 & hsa_circ_0040792 & 0.046578567 & 0.999852393 & 1.8944367 & chr16 & NM_017566 & KLHDC4 \\
\hline
\end{tabular}

we clustered all the different circRNAs to characterize the expression pattern of circRNA. The results are exhibited in Figure 4. Among them, red stand for elevated and green represents downregulated circRNAs.

3.2. $q P C R$ Verification. To verify differentially expressed circRNAs in AP, we made qPCR. And we selected three upregulated circRNAs including hsa_circRNA_101015, hsa_circRNA_101211, and hsa_circRNA_103470 and three downregulated circRNAs including hsa_circRNA_002532, hsa_circRNA_059665, and hsa_circRNA_104156. We validated the expression of three downregulated circRNAs in MAP and normal humans. And the results are shown in Figure 5. $P$ values are 0.008 (hsa_circRNA_002532), $<0.0001$ (hsa_circRNA_059665), and $<0.0001$

(hsa_circRNA_104156), respectively.

To further explore the relationship between the three upregulated circRNAs and disease severity, we compared the expression levels in healthy individuals, MAP, and SAP patients, respectively. As shown in Figure 6, we found that as the condition worsened, the expression levels of hsa_circRNA_101015 ( $P$ value was $0.0003,<0.0001$, and $<0.0001$, respectively), hsa_circRNA_101211 ( $P$ value was $0.0014,0.0478$, and $<0.0001$, respectively), and hsa_circ RNA_103470 ( $P$ value was $0.001,<0.0001$, and $<0.0001)$ also increased significantly. Then we validated the expression levels of three upregulated circRNAs in MAP patients and MAP after treatment. The results are demonstrated in Figure 7. After treatment, the expression levels of hsa_circRNA_101015, hsa_circRNA_101211, and hsa_circRNA_103470 in MAP were significantly declined $(P$ value $<0.0001)$.

3.3. ROC Analysis of hsa_circRNA_101015, hsa_circRNA_101211, and hsa_circRNA_103470 in Patients with AP. The ROC curve was constructed to assess the diagnostic significance of the three elevated circRNAs (Figure 8). RUCs of hsa_circRNA_101015, hsa_circ RNA_101211, and hsa_circRNA_103470 MAP patients with healthy people were 0.768 (95\% CI, 0.651-0.886, $P<0.001$, power $=0.97119), 0.731 \quad(95 \% \mathrm{CI}, 0.605-0.857, P=0.002$, power $=0.90168), 0.770 \quad(95 \% \mathrm{CI}, 0.653-0.887, P<0.001$, power $=0.97340)$, respectively. RUC of the three circRNAs combination was 0.838 (95\% CI, 0.738-0.937, $P<0.001$, power $=0.99954)$. The above results show that hsa_circRNA_101015, hsa_circRNA_101211, and hsa_circ RNA_103470 can be used as biomarkers to diagnose AP at early stage.

3.4. The Construction of ceRNA Network. Recent evidence suggests that circular RNA plays a crucial role in the regulation of miRNA-mediated gene expression regulation by isolating miRNAs. Their interaction with disease-associated miRNAs suggests that circular RNA is important for disease regulation [20]. To assess the potential function of circRNA, we investigated potential miRNAs that bind to circRNA. Then we constructed a ceRNA network for the six 




FIGURE 4: The hierarchical cluster of the differentially expressed circRNAs. 


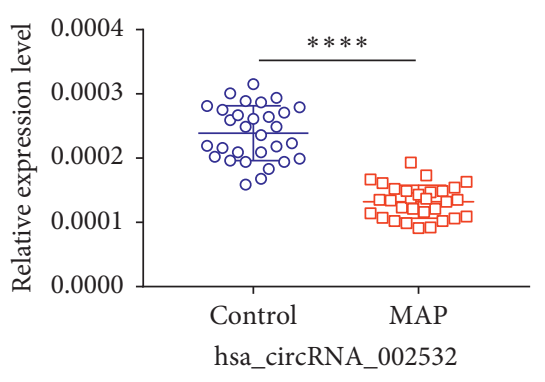

(a)

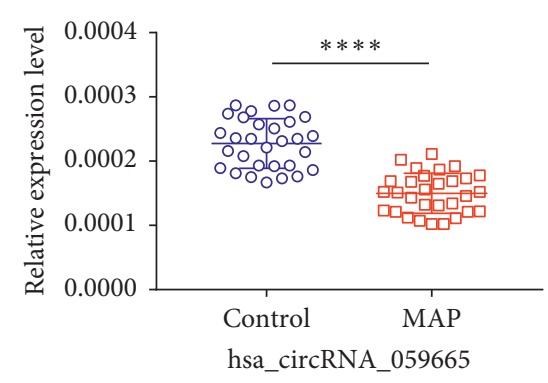

(b)



(c)

FiguRE 5: qPCR analysis of circRNA expression levels in MAP and healthy individuals including hsa_circRNA_002532, hsa_circRNA_059665, and hsa_circRNA_104156 $\left({ }^{*} P<0.05 ;{ }^{* * *} P<0.001 ;{ }^{* * * *} P<0.0001\right)$.

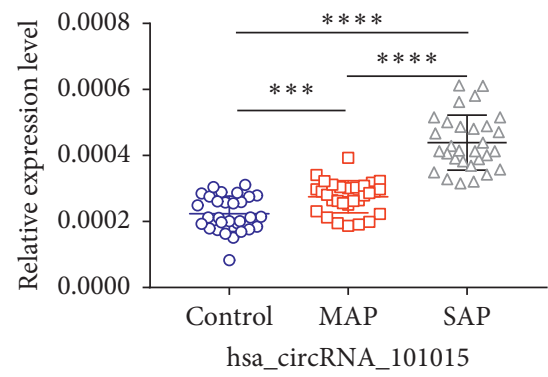

(a)

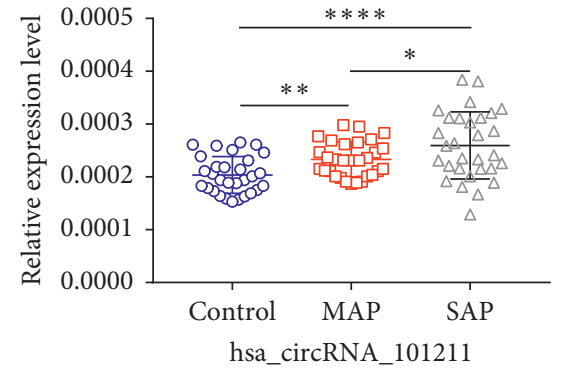

(b)

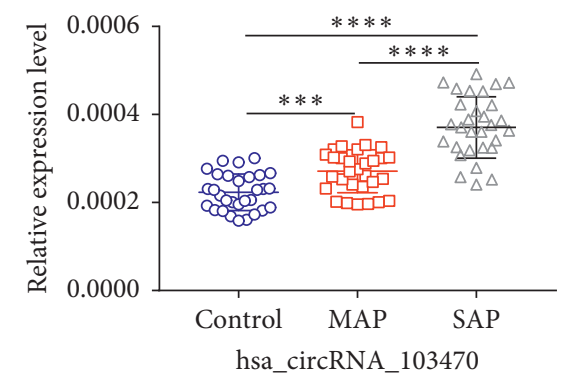

(c)

FIGURE 6: qPCR analysis of circRNA expression levels in healthy human, MAP, SAP patients including hsa_circRNA_101015, hsa_circRNA_101211, and hsa_circRNA_103470 $\left({ }^{*} P<0.05 ;{ }^{* * *} P<0.001 ;{ }^{* * * *} P<0.0001\right)$.

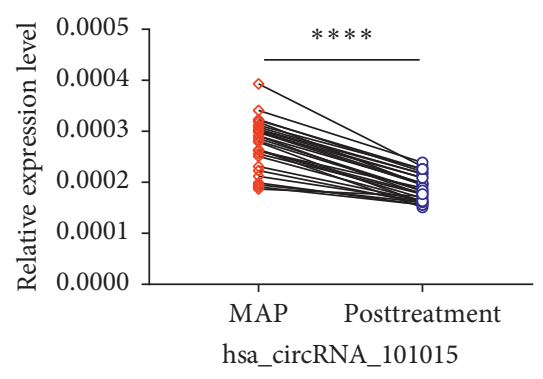

(a)

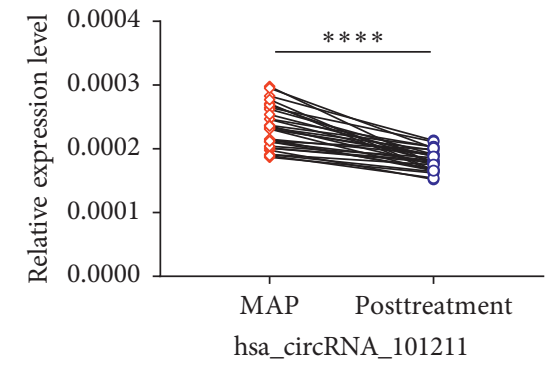

(b)

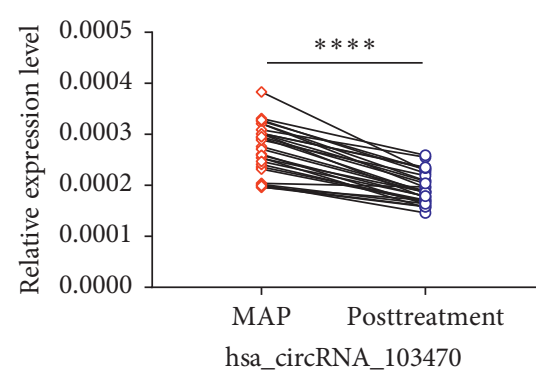

(c)

FIgURE 7: qPCR analysis of circRNA expression levels in MAP and MAP after treatment including hsa_circRNA_101015, hsa_circRNA_101211, and hsa_circRNA_103470 $\left({ }^{*} P<0.05 ;{ }^{* * *} P<0.001 ;{ }^{* * * *} P<0.0001\right)$.

differentially expressed circRNAs. In the ceRNA network, there are 241 nodes and 831 edges (Figure 9). Among them, for all nodes, red represents microRNAs, light-blue color represents protein_coding RNAs, and at the same time, brown color represents circular RNAs. Considering all edges, T-shape arrow represents directed relationships, while edges without arrow stand for undirected relationships. Specific details are shown in Supplementary Table 1.

\section{Discussion}

AP is an inflammatory process of the pancreas and has become an increasingly common clinical disease. In the second or third week of the disease, $40-70 \%$ of patients develop infectious necrosis and are the leading cause of late death [21]. In the United States, AP accounts for $\$ 2.5$ billion in medical expenses, and the number of hospital admissions is about 275,000 per year [22]. Because of the high mortality rate of $20-30 \%$ in severe cases of AP, early detection of patients who may need to be transferred to the intensive care unit (ICU) is critical. For patients with AP, accurate diagnosis, appropriate triage, high-quality supportive care, monitoring and treatment of complications, and prevention of recurrence are also critical [23]. The evaluation of biomarkers helps to further improve the identification of highrisk patients. In general, the clinical treatment decisions for AP depend primarily on the severity of the condition. MAP 


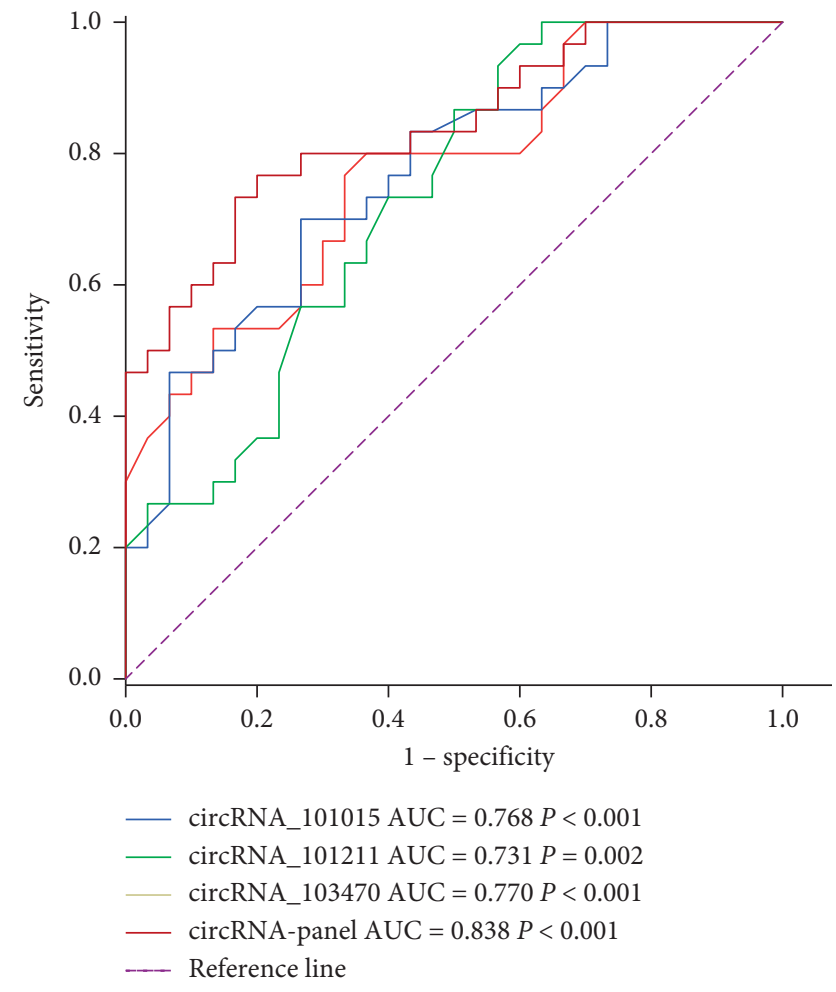

FIgURE 8: ROC analysis of hsa_circRNA_101015, hsa_circ RNA_101211,and hsa_circRNA_103470 in MAP patients versus control group.

is usually self-limiting and does not cause death. However, SAP can develop rapidly, leading to multiple organ failure and becoming life threatening. Therefore, the treatment of MAP patients is relatively simple and requires only a short hospitalization, while the treatment of SAP patients usually involves intensive care. Whether it is MAP or SAP, quick and accurate diagnosis is still very difficult.

Accurate diagnosis of AP may allow for effective treatment to begin earlier. At present, the accuracy of different scoring systems is not high, and a unique model is needed [24]. There are several laboratory tests such as blood urea nitrogen, creatinine, and hematocrit [25]. However, there is virtually no laboratory test that consistently and accurately predicts the severity of AP earlier [26, 27]. Many predictive systems use CT findings, but CT evidence of SAP lags behind clinical findings, and early CT studies may underestimate the severity of the disease. The scoring system is complex and cumbersome; therefore, these scoring systems are not a substitute for the clinician's experience assessment.

In recent years, circRNA has received wide attention as a new class of endogenous and regulatory noncoding RNAs. At the same time, with the widespread use of RNA sequencing (RNA-seq) technology and bioinformatics prediction, a large number of circRNAs have been identified. So far, no study has examined the role of circRNA in AP. Considering that circRNA is involved in a variety of diseases, it is necessary to explore differences in the expression of circRNA in patients with AP. Microarrays are an effective tool for analyzing circRNA. Therefore, in our study, we made full use of microarray technology and selected three patients with MAP and three healthy individuals. Our aim was to explore the differential expression of circRNA in the patients' blood with $\mathrm{AP}$ to diagnose AP patients as soon as possible. As a result, we found 25 upregulated circRNAs and 26 downregulated circRNAs. The vast majority of circRNAs have not been studied and require more in-depth exploration.

To further validate the six circRNAs in AP, we performed qPCR analysis. The results of qPCR studies provide novel biomarkers for molecular diagnosis and evaluation in AP. Three circRNAs including hsa_circRNA_002532 hsa_circRNA_059665, and hsa_circRNA_104156 were downregulated in MAP compared with healthy individuals. Three upregulated circRNAs including hsa_circRNA_101015, hsa_circRNA_101211, and hsa_circRNA_103470 significantly increased expression levels as the condition worsens. Furthermore, the expression levels of hsa_circRNA_101015, hsa_circRNA_101211, and hsa_circRNA_103470 were significantly reduced after treatment. This result suggests that the above three circRNAs may be involved in the development of AP and show promise as potential biomarkers for AP. To evaluate the specificity and sensitivity of the hsa_circRNA_101015, hsa_circRNA_101211, and hsa_circRNA_103470, we performed a ROC curve for further validation. The RUC was $0.768,0.731$, and 0.770 , respectively, and the combined RUC values of the three circRNAs were 0.838 . Therefore, the three circRNAs can be used as diagnostic biomarkers for AP with high sensitivity.

However, the pathogenesis of AP remains unknown. Therefore, the 6 differentially expressed circRNAs were selected to construct a ceRNA network including three upregulated hsa_circRNA_101015, hsa_circRNA_101211, and hsa_circ RNA_103470 and three downregulated hsa_circRNA_00 2532 hsa_circRNA_059665, and hsa_circRNA_104156. In the ceRNA network, we found a complex regulatory network for the above six circRNAs. However, the above six differentially expressed circRNAs excluding hsa_circRNA_104156 have not been reported. Considering that circRNAs can act as sponges of miRNAs, it is of importance to explore miRNAs in AP. More importantly, miRNAs play a critical role in the progression of AP. For example, a previous study found that miR-92b, miR10a, and miR-7 are downregulated in the blood of patients with $\mathrm{AP}$ and can be used to distinguish between patients with $\mathrm{AP}$ and healthy cases. In addition, the expression level of miR-551b-5p distinguishes between MAP and SAP [28]. However, these six circRNAs cannot interact with above these miRNAs. In the ceRNA network, hsa_circRNA_101015 could become sponges of several miRNAs such as hsa-miR-135a/b and hsa-miR-543. It has been reported that hsa-miR-135 was upregulated in the serum of mice with acute pancreatitis [29]. Furthermore, it has reported that hsa-miR-135a/b binding polymorphism can reduce the expression of $\mathrm{CD} 133$ and reduce the risk of lung cancer and improve the prognosis of patients [30]. Abnormalities in hsa-miR-543 can lead to increased proliferation of multiple cancers, such as esophageal cancer, prostate cancer, and osteosarcoma [31-33]. In the ceRNA network, hsa-miR-135 could be a target of hsa_circRNA_101015. It has been found that hsamiR-216a was downregulated in the serum of mice with acute 


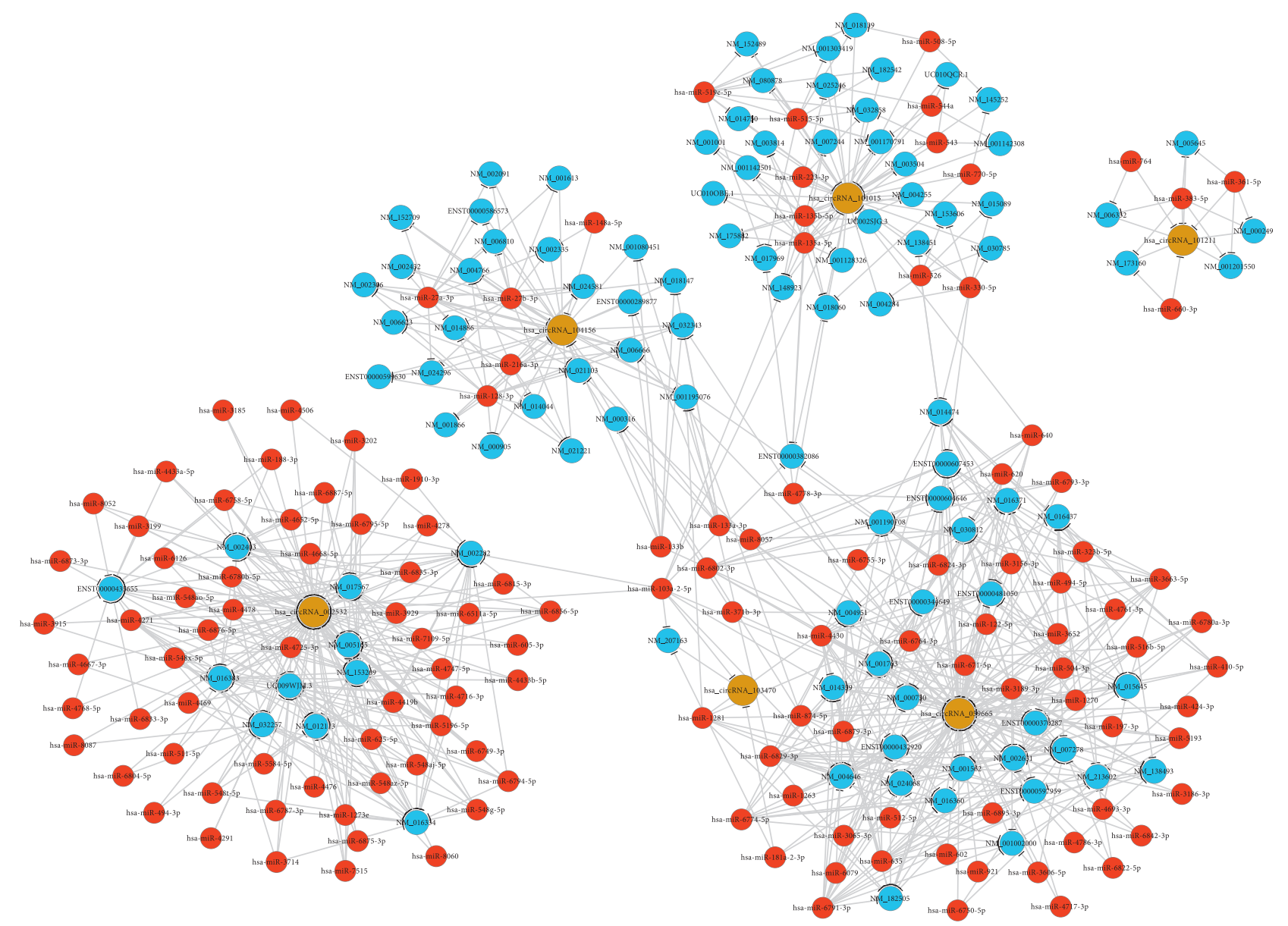

FIgure 9: The ceRNA network of 6 differentially expressed circRNAs including hsa_circRNA_101015; hsa_circRNA_103470; hsa_circRNA_059665; hsa_circRNA_002532; hsa_circRNA_101211; hsa_circRNA_104156.

pancreatitis [34]. Elevated serum hsa-miR-122 has been considered as a noninvasive marker for acute pancreatitis, which could become a target miRNA of hsa_circRNA_059665 as shown in the ceRNA network [35]. Moreover, hsa-miR-148a has been validated to be lowly expressed in acute pancreatitis [36]. Our results showed that hsa-miR-148a could be a potential target miRNA of hsa_circRNA_059665. Furthermore, hsa-miR148a could inhibit autophagy through IL-6/STAT3 axis in AP in acute pancreatitis. According to the ceRNA network, hsa_circ RNA_104156 could be considered as a sponge of hsa-miR-148a.

Therefore, we inferred that the six circRNAs could participate in pathogenic process of AP. However, specific research mechanisms need to be explored. In the future, we would continue to research the molecular mechanisms of hsa_circRNA_101015, hsa_circRNA_101211, and hsa_circ RNA_103470 in AP.

\section{Conclusion}

After microarray analysis and qPCR, we found three downregulated circRNAs including hsa_circRNA_002532, hsa_circRNA_059665, and hsa_circRNA_104156 and three upregulated circRNAs including hsa_circRNA_101015, hsa_circRNA_101211, and hsa_circRNA_103470 in the blood of AP patients. The ceRNA network revealed that the six circRNAs could play a critical role in AP. Therefore, elevated hsa_circRNA_101015, hsa_circRNA_101211, and hsa_circ RNA_103470 can be used as biomarkers in the blood to diagnose AP at early stage. The molecular mechanisms of elevated hsa_circRNA_101015, hsa_circRNA_101211, and hsa_circRNA_103470 in the pathological processes of AP would be explored in our further experiments.
Abbreviations
AP: $\quad$ Acute pancreatitis
circRNAs: Circular RNAs
qPCR: Quantitative polymerase chain reaction
ROC: Receiver operating characteristic
SAP: $\quad$ Severe acute pancreatitis
MAP: $\quad$ Mild acute pancreatitis
miRNAs: MicroRNAs
AUCs: Area under the ROC curves.

\section{Data Availability}

The data used to support the findings of this study are included within the supplementary information file. 


\section{Disclosure}

Chang Liu and Xuan Zhu are co-first authors.

\section{Conflicts of Interest}

The authors declare no conflicts of interest.

\section{Authors' Contributions}

Chang Liu and Xuan Zhu contributed equally to this article.

\section{Supplementary Materials}

Supplementary Table 1: the details of the ceRNA network. (Supplementary Materials)

\section{References}

[1] S. M. van Dijk, N. D. L. Hallensleben, H. C. van Santvoort et al., "Acute pancreatitis: recent advances through randomised trials," Gut, vol. 66, no. 11, pp. 2024-2032, 2017.

[2] P. A. Banks, M. L. Freeman, and Practice Parameters Committee of the American College of Gastroenterology, "Practice guidelines in acute pancreatitis," The American Journal of Gastroenterology, vol. 101, no. 10, pp. 2379-2400, 2006.

[3] C. D. Johnson and M. Abu-Hilal, "Persistent organ failure during the first week as a marker of fatal outcome in acute pancreatitis," Gut, vol. 53, no. 9, pp. 1340-1344, 2004.

[4] R. Mofidi, M. D. Duff, S. J. Wigmore, K. K. Madhavan, O. J. Garden, and R. W. Parks, "Association between early systemic inflammatory response, severity of multiorgan dysfunction and death in acute pancreatitis," British Journal of Surgery, vol. 93, no. 6, pp. 738-744, 2006.

[5] J. Kirkegård, D. Cronin-Fenton, U. Heide-Jørgensen, and F. V. Mortensen, "Acute pancreatitis and pancreatic cancer risk: a nationwide matched-cohort study in Denmark," Gastroenterology, vol. 154, no. 6, pp. 1729-1736, 2018.

[6] U. Ahmed Ali, Y. Issa, J. C. Hagenaars et al., "Risk of recurrent pancreatitis and progression to chronic pancreatitis after a first episode of acute pancreatitis," Clinical Gastroenterology and Hepatology, vol. 14, no. 5, pp. 738-746, 2016.

[7] D. Yadav, M. O'Connell, and G. I. Papachristou, "Natural history following the first attack of acute pancreatitis," American Journal of Gastroenterology, vol. 107, no. 7, pp. 1096-1103, 2012.

[8] P. G. Lankisch, M. Apte, and P. A. Banks, "Acute pancreatitis," The Lancet, vol. 386, no. 9988, pp. 85-96, 2015.

[9] O. Sadr-Azodi, Å. Andrén-Sandberg, N. Orsini, and A. Wolk, "Cigarette smoking, smoking cessation and acute pancreatitis: a prospective population-based study," Gut, vol. 61, no. 2, pp. 262-267, 2012.

[10] S. Stigliano, H. Sternby, E. de Madaria, G. Capurso, and M. S. Petrov, "Early management of acute pancreatitis: a review of the best evidence," Digestive and Liver Disease, vol. 49, no. 6, pp. 585-594, 2017.

[11] S. M. Staubli, D. Oertli, C. A. Nebiker et al., "Laboratory markers predicting severity of acute pancreatitis," Critical Reviews in Clinical Laboratory Sciences, vol. 52, no. 6, pp. 273-283, 2015.

[12] X. Li, L. Yang, and L.-L. Chen, "The biogenesis, functions, and challenges of circular RNAs," Molecular Cell, vol. 71, no. 3, pp. 428-442, 2018.
[13] J. Salzman, "Circular RNA expression: its potential regulation and function," Trends in Genetics, vol. 32, no. 5, pp. 309-316, 2016.

[14] T. B. Hansen, T. I. Jensen, B. H. Clausen et al., "Natural RNA circles function as efficient microRNA sponges," Nature, vol. 495, no. 7441, pp. 384-388, 2013.

[15] M. Bezzi, J. Guarnerio, and P. P. Pandolfi, "A circular twist on microRNA regulation," Cell Research, vol. 27, no. 12, pp. 1401-1402, 2017.

[16] D. Han, J. Li, H. Wang et al., "Circular RNA circMTO1 acts as the sponge of microRNA-9 to suppress hepatocellular carcinoma progression," Hepatology, vol. 66, no. 4, pp. 1151-1164, 2017.

[17] A. J. Enright, B. John, U. Gaul, T. Tuschl, C. Sander, and D. S. Marks, "MicroRNA targets in Drosophila," Genome Biology, vol. 5, no. 1, p. R1, 2003.

[18] A. E. Pasquinelli, "MicroRNAs and their targets: recognition, regulation and an emerging reciprocal relationship," Nature Reviews Genetics, vol. 13, no. 4, pp. 271-282, 2012.

[19] J.-H. Li, S. Liu, H. Zhou, L.-H. Qu, and J.-H. Yang, "starBase v2.0: decoding miRNA-ceRNA, miRNA-ncRNA and proteinRNA interaction networks from large-scale CLIP-Seq data," Nucleic Acids Research, vol. 42, no. D1, pp. D92-D97, 2014.

[20] S. Ghosal, S. Das, R. Sen, P. Basak, and J. Chakrabarti, "Circ2Traits: a comprehensive database for circular RNA potentially associated with disease and traits," Frontiers in Genetics, vol. 4, p. 283, 2013.

[21] P. A. Banks, T. L. Bollen, C. Dervenis et al., "Classification of acute pancreatitis-2012: revision of the Atlanta classification and definitions by international consensus," Gut, vol. 62, no. 1, pp. 102-111, 2013.

[22] A. Nieminen, M. Maksimow, P. Mentula et al., "Circulating cytokines in predicting development of severe acute pancreatitis," Critical Care, vol. 18, no. 3, p. R104, 2014.

[23] C. E. Forsmark, S. Swaroop Vege, and C. M. Wilcox, "Acute pancreatitis," The New England Journal of Medicine, vol. 375, no. 20, pp. 1972-1981, 2016.

[24] J. H. Cho, T. N. Kim, and H. H. Chung, "Comparison of scoring systems in predicting the severity of acute pancreatitis," World Journal of Gastroenterology, vol. 21, no. 8, pp. 2387-2394, 2015.

[25] C. E. Forsmark and D. Yadav, "Predicting the prognosis of acute pancreatitis," Annals of Internal Medicine, vol. 165, no. 7, pp. 523-524, 2016.

[26] S. Tenner, J. Baillie, J. DeWitt, and S. S. Vege, "American college of gastroenterology guideline: management of acute pancreatitis," The American Journal of Gastroenterology, vol. 108, no. 9, pp. 1400-1415, 2013.

[27] Working Group IAP/APA Acute Pancreatitis Guidelines, "IAP/APA evidencebased guidelines for the management of acute pancreatitis," Pancreatology, vol. 13, no. 4, pp. e1-e15, 2013.

[28] P. Liu, L. Xia, W.-l. Zhang et al., "Identification of serum microRNAs as diagnostic and prognostic biomarkers for acute pancreatitis," Pancreatology, vol. 14, no. 3, pp. 159-166, 2014.

[29] T. Qin, Q. Fu, Y.-f. Pan et al., "Expressions of miR-22 and miR-135a in acute pancreatitis," Journal of Huazhong University of Science and Technology [Medical Sciences], vol. 34, no. 2, pp. 225-233, 2014.

[30] M. Cheng, L. Yang, R. Yang et al., “A microRNA-135a/b binding polymorphism in CD133 confers decreased risk and favorable prognosis of lung cancer in Chinese by reducing CD133 expression," Carcinogenesis, vol. 34, no. 10, pp. 2292-2299, 2013. 
[31] H. Zhao, C. Diao, X. Wang et al., "MiR-543 promotes migration, invasion and epithelial-mesenchymal transition of esophageal cancer cells by targeting phospholipase A2 group IVA," Cellular Physiology and Biochemistry, vol. 48, no. 4, pp. 1595-1604, 2018.

[32] Y. Du, X.-h. Liu, H.-c. Zhu, L. Wang, J.-z. Ning, and C.-c. Xiao, "MiR-543 promotes proliferation and epithelialmesenchymal transition in prostate cancer via targeting RKIP," Cellular Physiology and Biochemistry, vol. 41, no. 3, pp. 1135-1146, 2017.

[33] L.-H. Wang, H.-C. Tsai, Y.-C. Cheng et al., "CTGF promotes osteosarcoma angiogenesis by regulating miR-543/angiopoietin 2 signaling," Cancer Letters, vol. 391, pp. 28-37, 2017.

[34] X.-X. Zhang, L.-H. Deng, W.-W. Chen et al., "Circulating microRNA 216 as a marker for the early identification of severe acute pancreatitis," The American Journal of the Medical Sciences, vol. 353, no. 2, pp. 178-186, 2017.

[35] P. Lu, F. Wang, J. Wu et al., "Elevated serum miR-7, miR-9, miR-122, and miR-141 are noninvasive biomarkers of acute pancreatitis," Disease Markers, vol. 2017, Article ID 7293459, 8 pages, 2017.

[36] B. Miao, W.-j. Qi, S.-w. Zhang et al., "miR-148a suppresses autophagy by down-regulation of IL-6/STAT3 signaling in cerulein-induced acute pancreatitis," Pancreatology, vol. 19, no. 4, pp. 557-565, 2019. 